Matteo Riccò ${ }^{1}$

Silvia Cattani ${ }^{2}$

Giovanni Gualerzi ${ }^{3}$

Carlo Signorelli ${ }^{4}$

\title{
WORK WITH VISUAL DISPLAY UNITS AND MUSCULOSKELETAL DISORDERS: A CROSS-SECTIONAL STUDY
}

\author{
PRACA PRZY MONITORZE EKRANOWYM A ZABURZENIA MIĘŚNIOWO-SZKIELETOWE - \\ BADANIE PRZEKROJOWE
}

\author{
${ }^{1}$ Provincial Agency for Health Services of the Autonomous Province of Trento, Trento, Italy \\ Department of Prevention, Occupational Health and Safety Unit \\ ${ }^{2}$ University of Parma, Parma, Italy \\ School of Nursing Sciences, Department of Clinical Surgery, General Surgery and Surgical Therapy \\ ${ }^{3}$ University of Parma, Parma, Italy \\ School of Medicine and Surgery, Department of Clinical and Experimental Medicine \\ ${ }^{4}$ University of Parma, Parma, Italy \\ Specialization School in Hygiene and Public Health, Department of Biomedical, Biotechnological and Translational Sciences
}

\begin{abstract}
Background: Epidemiological studies have shown that employees working with visual display units (VDU) are more likely to complain about musculoskeletal disorders (MSDs). The aim of this study has been to evaluate associations among MSDs and individuals and work-related factors. Material and Methods: A total of 1032 VDU workers were assessed about their personal (i.e., age, working history, smoking history, physical activity) and work-related factors (i.e., predominant job tasks performed, work posture). Work environment was evaluated regarding fulfillment of the standard ISO 9241-5:1998. The investigation required a direct observation of participants (in order to accurately assess the prevalence of MSDs) and workstations. Adjusted odds ratios $\left(\mathrm{OR}_{\mathrm{a}}\right.$ ) were calculated by means of the logistic regression model. Results: Prevalence of MSDs was relatively high $(53 \%)$. In general, MSDs were significantly associated with female sex (OR $=2.832$, 95\% confidence interval (CI): $2.178-3.683)$, age $\geq 50$ years old $(\mathrm{OR}=2.231,95 \% \mathrm{CI}: 1.236-4.026)$, longer exposure to VDU, both as working history (10-14 years: $\mathrm{OR}=1.934,95 \% \mathrm{CI}: 1.301-2.875 ; \geq 15$ years: $\mathrm{OR}=2.223,95 \% \mathrm{CI}: 1.510-3.271)$ and working time $(30-39 \mathrm{~h} /$ week: $\mathrm{OR}=1.537,95 \% \mathrm{CI}: 1.087-2.273$ ). Inappropriate workstation design was confirmed by the multivariate analysis as a risk factor for MSDs (OR = 2.375, 95\% CI: 1.124-5.018). Conclusions: Musculoskeletal disorders were significantly associated with individual factors as well as characteristics of work environment. An appropriate design of workstations may significantly reduce their prevalence amongst VDU workers. Med Pr 2016;67(6):707-719
\end{abstract}

Key words: job stress, ergonomics, work-related musculoskeletal disorders, musculoskeletal disorders, office workers, visual display unit

\section{STRESZCZENIE}

Wstęp: Badania epidemiologiczne wykazały, że osoby pracujące przy monitorach ekranowych (visual display units - VDU) częściej skarżą się na zaburzenia mięśniowo-szkieletowe (musculoskeletal disorders - MSDs). Celem badania była ocena związku między MSDs a cechami indywidualnymi pracowników i czynnikami związanymi z pracą. Materiał i metody: Badanie przeprowadzono wśród 1032 osób używających VDU w pracy. Zebrano dane dotyczące cech indywidualnych badanych (wiek, staż pracy, historia palenia, aktywność fizyczna) i czynników związanych z wykonywaną pracą (najczęstsze czynności, pozycja ciała). Oceniono też zgodność środowiska pracy z normą ISO 9241-5:1998. W celu dokładnej analizy występowania MSDs przeprowadzono bezpośrednią obserwację osób badanych i ich stanowisk pracy. Skorygowane ilorazy szans (adjusted odds ratios - OR ) obliczono z zastosowaniem modelu regresji logistycznej. Wyniki: Częstość występowania MSDs była stosunkowo wysoka (53\%). Zaobserwowano statystycznie istotną zależność między MSDs a płcią żeńską (OR = 2,832, 95\% przedział ufności (confidence interval - CI): 2,178-3,683), wiekiem powyżej 50 lat (OR = 2,231, 95\% CI: 1,236-4,026) i dłuższym narażeniem na VDU - zarówno w wyniku dłuższego stażu pracy (10-14 lat: OR = 1,934, 95\% CI: 1,301-2,875; $\geq 15$ lat: OR = 2,223, 95\% CI: 1,510-3,271), jak i większego wymiaru czasu pracy (30-39 godz./tydzień: OR = 1,537, 95\% CI: 1,087-2,273). Analiza wieloczynnikowa potwierdziła, że nieodpowiednio zorganizowane stanowisko pracy jest czynnikiem ryzyka MSDs (OR = 2,375, 95\% CI: 1,124-5,018). Wnioski: Występowanie zaburzeń mięśniowo-szkieletowych jest istotnie związane z indywidualnymi cechami pracownika i właściwościami środowiska pracy. Odpowiednio zorganizowane stanowiska pracy mogą znacznie zmniejszyć częstość występowania MSDs u osób pracujących przy VDU. Med. Pr. 2016;67(6):707-719

Słowa kluczowe: stres w pracy, ergonomia, zaburzenia mięśniowo-szkieletowe związane z pracą, zaburzenia mięśniowo-szkieletowe, pracownicy biurowi, monitor ekranowy 
Corresponding author / Autor do korespondencji: Matteo Riccò, Provincial Agency for Health Services of the Autonomous

Province of Trento, Department of Prevention, Occupational Health and Safety Unit, Via Verona SNC,

C/O Centro Servizi Sanitari, 38123 Trento, Italy, e-mail: mricco2000@gmail.com, matteo.ricco@apss.tn.it

Received: February 13, 2016, accepted: July 27, 2016

\section{INTRODUCTION}

Computer use in the office environment has intensified in developed and developing countries, and it has been linked with high prevalence of complaints about neck, upper (i.e., fingers, hands, wrists, elbows, arms, shoulders) and lower extremities, low back/sacrum, and in particular with musculoskeletal disorders (MSDs) [1-4]. Work-related MSDs (WMSDs) may be defined as a diverse set of conditions characterized by pain, aching, stiffness, fatigue, discomfort, tingling and/or numbness, and may represent an impairment of body structures rather associated with than simply caused by cumulative exposure to work and working environment [5].

Often underestimated as a cause of occupational illness and sick leave, has it been estimated that up to $12 \%$ of the computer workers indicate productivity reductions due to discomfort, which results in $10-20 \%$ decrease in perceived productivity whereas absenteeism and medical expenses related to WMSDs in visual display unit (VDU) users may cost the industry 45-54 billion dollars annually, only in the USA [6]. Despite the fact that similar estimates for the European Union (EU) countries are not available, several crosssectional studies performed in Europe have reported a prevalence of $30-62 \%$ of musculoskeletal symptoms in the neck or shoulder region studies, and suggest that referred complaints would increase with age and working age, being more prevalent among women than men [4,6-12]. However, as this same difference has been identified in the general working population, its causative association may be doubtful [13-15].

In facts, the aetiology of WMSDs in VDU workers is not completely understood but there is some evidence that office workers are significantly exposed to physical (i.e., repetitive movement, awkward and/or static postures of the arm and neck, and manual handling tasks) and psychological (i.e., time pressures, high quantitative job demands and limited control over the work's content, limited support from co-workers/supervisors, stressful work) risk factors [10,16-20].

However, several caveat should be addressed. First of all, musculoskeletal pain is the most frequently reported health problem in general as well as in the working pop- ulation [1-5,13]. Not coincidentally, are WMSDs, and particularly neck and back pain and upper-limb symptoms therefore interpreted as common health problems with a multifactorial aetiology, including mixed, prolonged and often ill-defined exposures over a long period of time [5-7,10-11]. Moreover, as a large number of epidemiological studies about WMSDs amongst VDU workers are based on self-referred symptoms, the lack of clinical evaluation data, the possible risk is the overreporting of musculoskeletal complaints inconsistently associated [1,8,10-13]. Besides, studies about WMSDs often lack accurate evaluation of personal medical history, failing to refer to previous acute traumas or systemic diseases $[13,21]$. In other words, not only occupational but also personal history and individual risk factors (including age, gender, education level, smoking habits, etc.) influence the natural history of such disorders in probabilistic terms, both inducing and anticipating its clinical presentation $[3,11]$.

The objective of this study has therefore been to clinically investigate the epidemiology of MSDs in a study population of VDU workers, eventually assessing whether personal and occupational risk factors may be associated with the MSDs diagnosis.

\section{MATERIAL AND METHODS}

\section{Study group}

This was a cross-sectional study performed in 4 private companies based in Northern Italy in 2012-2013, as a part of a larger musculoskeletal survey performed as a part of the compulsory medical surveillance (Italian Legislative Decree No. 81, April 9, 2008 [22]). The study population initially encompassed a total of 1329 office workers who used VDU for more than $1 \mathrm{~h} /$ day. One aim of the study was to estimate the prevalence of MSDs associated with VDU use:

subjects referring to any previous exposure to tasks requiring weight lifting, repetitive movements of the upper limb(s), or vibrations (either whole body and upper limb);

subjects suffering from musculoskeletal diseases, such as rheumatoid arthritis, osteoarthritis and other disorders of the connective tissue; 
subjects having a personal history, including trauma and/or surgery of the neck/back/upper musculoskeletal extremity, preceding the exposure to VDU work, were excluded from the study $(\mathrm{N}=195)$.

Out of the remaining 1134 subjects, 1032 (91\%) corresponded to the inclusion criteria and were asked to participate in the study, eventually signing informed consent.

\section{Questionnaire assessment}

A structured questionnaire was administered to all participants in order to standardize data collection about demographic characters (gender, age, height and weight, formal education, occupational history, duration of employment), lifestyles (smoking history, physical exercise), pain condition, medical history. Regular physical activity was defined following the World Health Organization (WHO) recommendation for 18-64-year-olds, i.e., at least 150 min of moderateintensity aerobic physical activity throughout the week or at least $75 \mathrm{~min}$ of vigorous physical activity throughout the week or an equivalent combination of moderate- and vigorous-intensity activity [23].

Participants were then asked about their job, both in general (i.e., hours of VDU exposure during the working week; free or externally managed schedule of rest breaks) and more specifically about assignments (i.e., front office vs. back office) and contents: job content was defined by the predominant (i.e., performed for $>50 \%$ of the shift) task as "word processing," "data entry," "data processing" or "programming," the latter in general including all activities leading from an original formulation to an executable program. Where a predominant task was not identified, the job content was arbitrarily defined as a "various" one.

Eventually, satisfaction of participants towards their job was assessed through a 4-point Likert scale (i.e., very dissatisfied, dissatisfied, satisfied, highly satisfied).

\section{Workplace assessment}

Workstations were directly assessed by researchers regarding the predominant position (i.e., sitting or alternate sit-standing) then focusing on the fulfillment of the International Organization for Standardization (ISO) standard 9241-5:1998 [24]. Requirements for desktops (height: $65-74 \mathrm{~cm}$, depth: $80-110 \mathrm{~cm}$, distance from the seat: $20-26 \mathrm{~cm}$ ), seats (adjustable chair height: $42-51 \mathrm{~cm}$ and depth: $40-42 \mathrm{~cm}$, adjustable backrest with height of 20-26 cm, 5-leg base with casters), and footrests (width $>40 \mathrm{~cm}$, height $4-15 \mathrm{~cm}$, inclination $0-15^{\circ}$ ) were specifically evaluated [24]. Results of the survey were univocally linked with the participant assigned to the specific workstation. Subjective elements were also inquired, as participants were asked whether they felt comfortable or not at their work station as a whole, subsequently detailing the perceived comfort for a seat and desktop.

\section{Collection of symptoms}

The Ergonomics of Posture and Movement (EPM) research unit medical questionnaire was compiled by the occupational physician, collecting pain, aches or discomfort in the back, neck and shoulders [25]. The questionnaire was previously validated as it had been found appropriate for use in Italian working population, and was commonly used as a musculoskeletal anamnestic utility by the occupational physician in Italy. All patients received a physical examination with specific attention to musculoskeletal signs and symptoms. Eventually, subjects were defined as positive for musculoskeletal disorders (MSDs) in the case of:

referring discomfort in the back (the neck with or without radiation into the leg to below to knee) for at least 1 day during the preceding 12 months, with/without pain elicited by palpation of paravertebral muscles and/or spinal apophyses, with/without positivity of direct and/or indirect Lasègue sign; referring persistent pain in upper arm districts (shoulder, elbow, wrist/hand) lasting at least 1 week during the preceding 12 months.

\section{Ethics}

The study was performed as a part of a compulsory health assessment of the workplace: the procedures were performed only in order to fully assess the clinical status and the workers' capability to work, and would be performed even when the study is not conducted. Therefore, no preliminary evaluation by the Ethical Committee was necessary. However, as clinical and personal particulars had been collected and elaborated, all participants gave their written consent and subjects refusing their consent were excluded from the study population.

\section{Statistical analysis}

Continuous variables were compared using the t-test whereas the $\mathrm{Chi}^{2}$ test was used for comparing categorical variables. The univariate analysis was used for calculating the odds ratios (ORs) and the 95\% confidence intervals (CI). Adjusted odds ratios $\left(\mathrm{OR}_{\mathrm{a}}\right)$ for categori- 
cal variables associated with musculoskeletal complaints for the purpose of the univariate analysis with $\mathrm{p}<0.15$ were calculated by means of a logistic regression model. The model included sex, age, and working age. In all statistical analyses, $\alpha$ was set at $\mathrm{p}<0.05$. All calculations were performed by using SPSS version 22.0 (IBM Corporation, United States).

\section{RESULTS}

\section{Demographics}

The mean age ( \pm standard deviation) of the 1032 participants, 375 (36.3\%) males and 657 (63.7\%) females, was $43.2 \pm 10.3$ years old, with 583 subjects $(56.5 \%)$ older than 40 years old. Not surprisingly, education level was relatively high, with $696(67.4 \%)$ subjects referring to a university or post-high school degree. Lifestyle assessment identified a current or past smoking history most frequently amongst females (31.8\%) rather than amongst males $(20.3 \%, \mathrm{p}<0.001)$ whereas a regular physical activity was referred to by 297 (28.8\%) subjects, similarly more frequently reported by females (30.7\%) than males $(25.3 \%, \mathrm{p}=0.076)$ (Table 1$)$.

The mean working age of the sample was $11.9 \pm 6.8$ years old, with $152(14.7 \%)$ participants referring to 1-4 years, 212 (20.5\%) participants - 5-9 years, 303 (29.4\%) participants - 10-14 years, and eventually 365 (35.4\%) participants having 15 or longer period of working history.

\section{Workplace and job assessment}

Focusing on the labour characteristics, 299 (29\%) participants worked fewer than $20 \mathrm{~h} /$ week with VDU, 460 (44.6\%) participants - between 20-29 h/week, $235(22.8 \%)$ participants - between 30-39 h/week, and eventually 38 (3.7\%) subjects reported to have been working more than $40 \mathrm{~h} /$ week. Management of rest breaks (i.e., at least 15 min every 120 min of continuous VDU use) was defined as free (i.e., autonomous) in the case of 897 (86.9\%) participants whereas in the case of remain-

Table 1. Demographic, work and lifestyle characteristics of the office workers studied in Italy, 2012-2013

Tabela 1. Czynniki demograficzne oraz związane z pracą i stylem życia pracowników biurowych badanych we Włoszech w latach 2012-2013

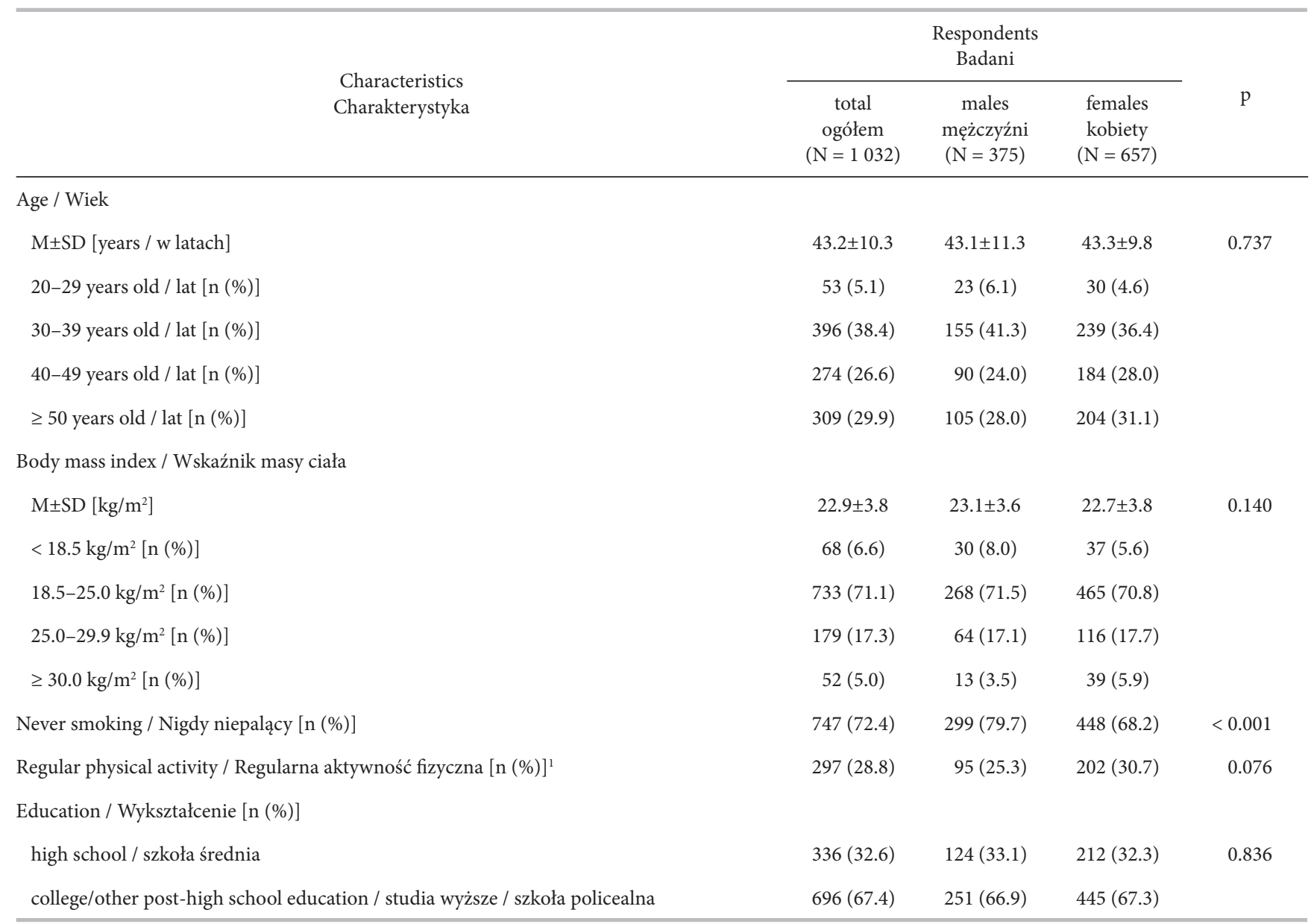


Table 1. Demographic, work and lifestyle characteristics of the office workers studied in Italy, 2012-2013 - cont.

Tabela 1. Czynniki demograficzne oraz związane z pracą i stylem życia pracowników biurowych badanych we Włoszech w latach 2012-2013 - cd.

Characteristics

Charakterystyka
Respondents

Badani

\begin{tabular}{|c|c|c|c|}
\hline $\begin{array}{c}\text { total } \\
\text { ogółem } \\
(\mathrm{N}=1032)\end{array}$ & $\begin{array}{c}\text { males } \\
\text { mężczyźni } \\
(\mathrm{N}=375)\end{array}$ & $\begin{array}{c}\text { females } \\
\text { kobiety } \\
(\mathrm{N}=657)\end{array}$ & $\mathrm{p}$ \\
\hline
\end{tabular}

Seniority / Staż pracy

$\mathrm{M} \pm \mathrm{SD}$ [years / w latach]

$11.5 \pm 6.7$

$12.1 \pm 6.8$

0.167

$1-4$ years / lat $[\mathrm{n}(\%)]$

5-9 years / lat [n (\%)]

$152(14.7)$

59 (15.7)

$93(14.2)$

$212(20.5)$

$86(22.9)$

$126(19.2)$

10-14 years / lat [n (\%)]

$303(29.4)$

$107(28.5)$

$196(29.8)$

$365(35.4)$

$123(32.8)$

$242(36.8)$

Free management of rest breaks / Przerwy na odpoczynek ustalane przez pracownika [n (\%)]

$897(86.9)$

$328(87.5)$

$569(86.6)$

0.693

Working time with computer / Wymiar czasu pracy przy komputerze

$\mathrm{M} \pm \mathrm{SD}$ [h/week / godz./tydzień]

$\begin{array}{ccc}22.3 \pm 8.6 & 22.9 \pm 10.3 & 22.0 \pm 7.4 \\ 299(29.0) & 118(31.5) & 181(27.5) \\ 460(44.6) & 145(41.1) & 315(47.9) \\ 235(22.8) & 82(21.9) & 153(23.3) \\ 38(3.7) & 30(8.0) & 8(1.2)\end{array}$

$\geq 40 \mathrm{~h} /$ week / godz./tydzień [n (\%)]

$38(3.7)$

$684(66.3)$

$250(66.7)$

$434(66.1)$

$<0.001$

$74(7.2)$

10 (2.7)

$64(9.7)$

$37(3.6)$

$13(3.5)$

$24(3.7)$

$192(18.6)$

$64(17.2)$

$128(19.5)$

$45(4.4)$

$38(10.1)$

7 (1.1)

$548(53.1)$

$150(40.0)$

$398(60.6)$

$<0.001$

975 (94.5)

353 (94.1)

$622(94.7)$

0.715

Workstation in accordance with ISO 9241-5:1998 / Stanowisko pracy zgodne z ISO 9241-5:1998 [n (\%)]

desktop / biurko

$870(84.3)$

$327(87.2)$

$543(82.6)$

0.053

$884(85.7)$

$310(82.7)$

$574(87.4)$

0.038

footrest / podnóżek

$772(74.8) \quad 332(88.5)$

$440(67.0)$

$<0.001$

$555(53.8)$

$243(64.8)$

$312(47.5)$

$<0.001$

Subjective comfort at workplace / Subiektywna ocena wygody stanowiska pracy [n (\%)]

$$
\text { desktop / biurko }
$$$$
889(86.1)
$$$$
335(89.3)
$$$$
554 \text { (84.3) }
$$$$
0.025
$$

334 (89.1)

$577(87.8)$

0.550

total / ogółem

$855(82.8)$

$319(85.1)$

536 (81.6)

0.153

471 (45.6)

$180(48.0)$

$291(44.3)$

0.250

M - mean / średnia, SD - standard deviation / odchylenie standardowe.

${ }^{1}$ At least $150 \mathrm{~min}$ of moderate-intensity aerobic physical activity throughout the week or at least $75 \mathrm{~min}$ of vigorous-intensity activity or an equivalent combination of moderate- and vigorous-intensity activity / Przynajmniej 150 min średnio intensywnych aerobowych ćwiczeń fizycznych w tygodniu lub 75 min intensywnych ćwiczeń, lub równoważna kombinacja średnio intensywnych i intensywnych ćwiczeń.

a Desktop - height: $65-74 \mathrm{~cm}$, depth: $80-110 \mathrm{~cm}$, distance from the seat: $20-26 \mathrm{~cm}$; seat - adjustable chair: $42-51 \mathrm{~cm}$ in height, $40-42 \mathrm{~cm}$ in depth, adjustable backrest: 20-26 cm in height, 5-leg base with casters; footrest - width: > $40 \mathrm{~cm}$, height: $4-15 \mathrm{~cm}$, inclination: 0-15² [24] / Biurko - wysokość: $65-74 \mathrm{~cm}$, głębokość: 80-110 cm, odległość od krzesła: $20-26 \mathrm{~cm}$; krzesło - wysokość regulowana: $42-51 \mathrm{~cm}$, głębokość: $40-42 \mathrm{~cm}$, wysokość regulowanego oparcia: $20-26 \mathrm{~cm}$, podstawa z 5 nogami i kółkami samonastawnymi; podnóżek - szerokość: $>40 \mathrm{~cm}$, wysokość: $4-15 \mathrm{~cm}$, nachylenie: $0-15^{\circ}$ [24]. 
ing 135 subjects (13.1\%) pauses were externally managed by a supervisor or time-planned during the working shift.

Daily tasks with VDU of participants mainly included content word processing (18.6\%), data entry (7.2\%), data processing (3.6\%), programming (4.4\%) whereas the large majority of the sample described the job content as a mixed activity encompassing 2 or more of the aforementioned tasks (66.3\%). For around a half of the subjects (53.1\%), job assignments included front office activity. More than a half of participants (53.4\%) were somehow unsatisfied with the current job assignment, with $6 \%(\mathrm{~N}=62)$ of the sample referring to a high degree of dissatisfaction. In general, current job assignment was defined as unsatisfying as "non intellectually stimulant" (76 out of 551, 13.8\%), "monotonous" ( $\mathrm{N}=49,8.9 \%)$, or "not interesting" $(\mathrm{N}=16,2.9 \%)$, whereas 33 participants complained the job assignments as "too intense" $(\mathrm{N}=33,6 \%)$.

Among the participants, $94.5 \%$ declared to perform office activities in a sitting position whereas 57 subjects (5.5\%) identified standing position as the more prevalent one. The standard ISO 9241-5:1998 [24] requirements were fulfilled by $84.3 \%$ of desktops, $88.3 \%$ of the seats, with $74.8 \%$ of workstations having appropriate footrests. Eventually, 555 out of 1032 (53.8\%) workstations appeared as fulfilling standard requirements (Table 1). Subjective assessment of workplace by participants exhibited similar figures, with $85.7 \%$ and $86.1 \%$ of participants respectively referring their seats and desktops as comfortable for job assignments (Pearson's $r=0.567$ and 0.160 , respectively, $\mathrm{p}<0.001$ in both cases) whereas $82.8 \%$ of participants were globally satisfied by workstation's comfort (Pearson's $\mathrm{r}=0.161, \mathrm{p}<0.001$ ).
In general, MSDs were identified in the case of 547 participants (53\%). Most frequently reported sites were neck (38.1\%), low back (29.1\%), and shoulders $(24.8 \%)$ whereas in the case of 109 (10.6\%) subjects and $86(8.3 \%)$ subjects a positive status was identified for elbow and hand/wrist, respectively (Figure 1). Prevalence of complaints increased through age groups, and eventually peaking for $63.1 \%$ of subjects $\geq 50$-year-old (195 out of 309) (Figure 2).

In the case of the univariate analysis, MSDs were significantly associated with the following personal factors (Table 2): female sex $(\mathrm{OR}=2.832$, 95\% CI: 2.178-3.683),

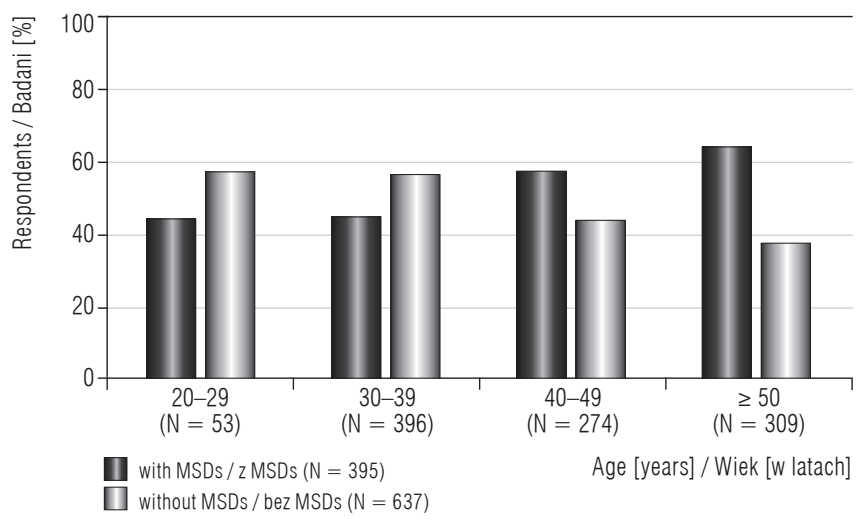

Prevalence of MSDs increased from 43.4\% among 20-29-year-old respondents to $43.9 \%$ among $30-39$-year-old respondents, $56.6 \%$ among $40-49$-year-old respondents and $63.1 \%$ among respondents $\geq 50$-year-old / Częstość występowania MSDs wzrastała z 43,4\% wśród osób w wieku 20-29 lat do 43,9\% wśród osób w wieku 30-39 lat, do 56,6\% wśród osób w wieku 40-49 lat i 63,1\% u osób $\geq 50$ lat.

Fig. 2. Musculoskeletal disorders (MSDs) of the office workers studied in Italy in years 2012-2013, by age

Ryc. 2. Zaburzenia mięśniowo-szkieletowe (MSDs) u pracowników biurowych badanych we Włoszech w latach 2012-2013 - według wieku

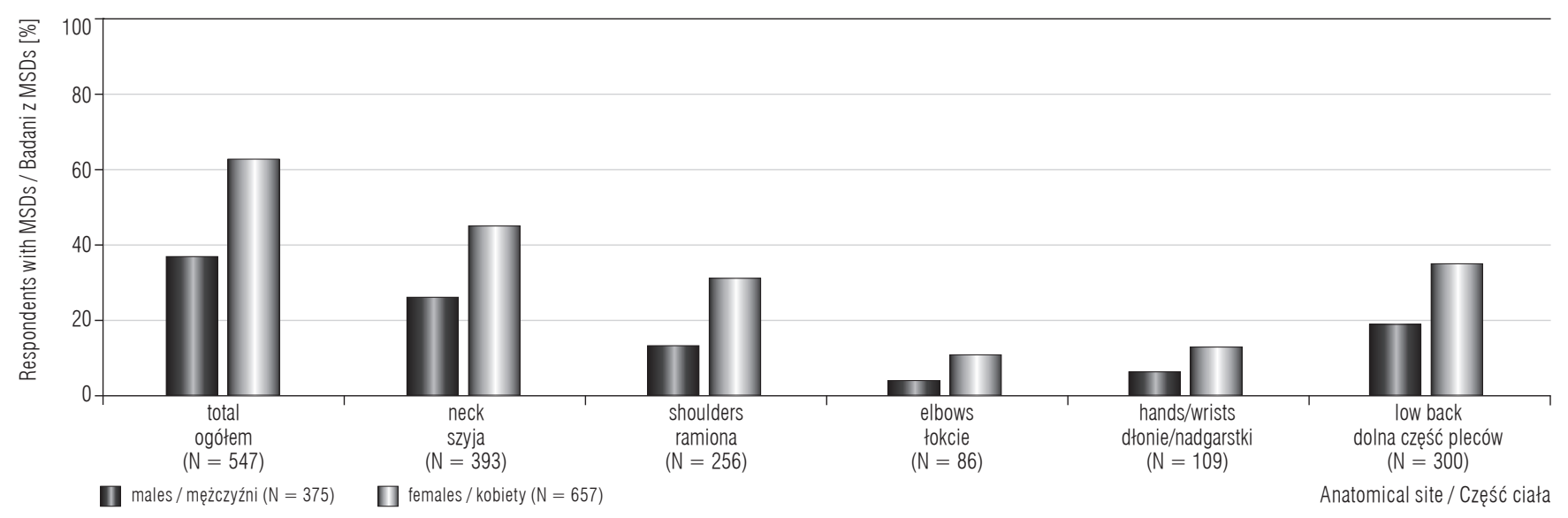

MSDs cases among studied respondents: 36.8\% males vs. 62.3\% females (p < 0.001) / Przypadki MSDs u badanych: 36,8\% mężczyzn vs 62,3\% kobiet (p < 0,001).

Fig. 1. Musculoskeletal disorders (MSDs) in the office workers studied in Italy, 2012-2013, by anatomical site and sex Ryc. 1. Zaburzenia mięśniowo-szkieletowe (MSDs) u pracowników biurowych badanych we Włoszech w latach 2012-2013 według części ciała i płci 
Table 2. Univariate analysis for musculoskeletal disorders (MSDs) dependent on demographics, lifestyle and work characteristics of office workers studied in Italy, 2012-2013

Tabela 2. Jednoczynnikowa analiza zaburzeń mięśniowo-szkieletowych (MSDs) zależnych od czynników demograficznych oraz związanych z pracą i stylem życia pracowników biurowych badanych we Włoszech w latach 2012-2013

\begin{tabular}{|c|c|c|c|c|c|}
\hline \multirow{2}{*}{$\begin{array}{l}\text { Characteristics } \\
\text { Charakterystyka }\end{array}$} & \multicolumn{2}{|c|}{$\begin{array}{c}\text { Respondents } \\
\text { Badani } \\
(\mathrm{N}=1032)\end{array}$} & \multirow{2}{*}{$\mathrm{p}$} & \multirow{2}{*}{ OR } & \multirow{2}{*}{$95 \% \mathrm{CI}$} \\
\hline & $\begin{array}{c}\text { total } \\
\text { ogółem } \\
{[\mathrm{n}]}\end{array}$ & $\begin{array}{l}\text { with MSDs } \\
\text { z MSDs } \\
\text { [n (\%)] }\end{array}$ & & & \\
\hline \multicolumn{6}{|l|}{ Sex / Płeć } \\
\hline females / kobiety & 657 & $409(62.3)$ & & 2.832 & $2.178-3.683$ \\
\hline \multicolumn{6}{|l|}{ Age / Wiek } \\
\hline $20-29$ years old / lat & 53 & $23(43.4)$ & ref. & 1.000 & - \\
\hline 30-39 years old / lat & 396 & $174(43.9)$ & 0.940 & 1.022 & $0.573-1.823$ \\
\hline \multicolumn{6}{|l|}{ Body mass index / Wskaźnik masy ciała } \\
\hline$>18.5 \mathrm{~kg} / \mathrm{m}^{2}$ & 67 & $21(31.3)$ & ref. & 1.000 & - \\
\hline $18.5-25.0 \mathrm{~kg} / \mathrm{m}^{2}$ & 733 & $451(61.5)$ & $<0.001$ & 3.503 & $2.047-5.995$ \\
\hline $25.0-29.9 \mathrm{~kg} / \mathrm{m}^{2}$ & 180 & $56(31.1)$ & 0.972 & 0.989 & $0.540-1.812$ \\
\hline$\geq 30.0 \mathrm{~kg} / \mathrm{m}^{2}$ & 52 & $19(36.5)$ & 0.552 & 1.261 & $0.587-2.710$ \\
\hline \multicolumn{6}{|l|}{ Smoking history / Historia palenia } \\
\hline never smoker / nigdy niepalący & 747 & $405(54.2)$ & 0.206 & 1.000 & - \\
\hline current/past smoker / palący obecnie lub w przeszłości & 285 & $142(49.8)$ & & 0.893 & $0.638-1.102$ \\
\hline $\begin{array}{l}\text { college/other post-high school education / studia } \\
\text { wyższe / szkoła policealna }\end{array}$ & 696 & $378(54.3)$ & & 1.175 & $0.905-1.524$ \\
\hline \multicolumn{6}{|l|}{ Seniority / Staż pracy } \\
\hline $1-4$ years / lat & 152 & $60(39.5)$ & ref. & 1.000 & - \\
\hline $5-9$ years / lat & 212 & $102(48.1)$ & 0.102 & 1.422 & $0.932-2.169$ \\
\hline $10-14$ years / lat & 303 & $169(55.8)$ & 0.001 & 1.934 & $1.301-2.875$ \\
\hline$\geq 15$ years / lat & 365 & $216(59.2)$ & $<0.001$ & 2.223 & $1.510-3.271$ \\
\hline \multicolumn{6}{|l|}{$\begin{array}{l}\text { Working time with computer / Wymiar czasu pracy przy } \\
\text { komputerze }\end{array}$} \\
\hline$<20$ h/week / godz./tydzień & 299 & $149(49.8)$ & ref. & 1.000 & - \\
\hline 20-29 h/week / godz./tydzień & 460 & $240(52.2)$ & 0.528 & 1.098 & $0.821-1.470$ \\
\hline 30-39 h/week / godz./tydzień & 235 & $142(60.4)$ & 0.015 & 1.537 & $1.087-2.173$ \\
\hline$\geq 40 \mathrm{~h} /$ week / godz./tydzień & 38 & $16(42.1)$ & 0.371 & 0.732 & $0.370-1.449$ \\
\hline
\end{tabular}

${ }^{1}$ As in Table $1 /$ Jak w tabeli 1.

OR - odds ratio / iloraz szans, CI - confidence interval / przedział ufności, ref. - reference group / grupa referencyjna. 
Table 3. Prevalence of musculoskeletal disorders (MSDs) of the office workers studied in Italy, 2012-2013, and objective and subjective assessment of their workstations

Tabela 3. Występowanie zaburzeń mięśniowo-szkieletowych (MSDs) u pracowników biurowych badanych we Włoszech w latach 2012-2013 a obiektywna i subiektywna ocena ich stanowisk pracy

\begin{tabular}{|c|c|c|c|c|c|}
\hline \multirow{2}{*}{$\begin{array}{l}\text { Variable } \\
\text { Zmienna }\end{array}$} & \multicolumn{2}{|c|}{$\begin{array}{c}\text { Respondents } \\
\text { Badani } \\
(\mathrm{N}=1032)\end{array}$} & \multirow[b]{2}{*}{$\mathrm{p}$} & \multirow{2}{*}{ OR $(95 \% \mathrm{CI})$} & \multirow[b]{2}{*}{$\mathrm{OR}_{\mathrm{a}}(95 \% \mathrm{CI})^{*}$} \\
\hline & $\begin{array}{l}\text { total } \\
\text { ogółem } \\
{[\mathrm{n}]}\end{array}$ & $\begin{array}{l}\text { with MSDs } \\
\text { z MSDs } \\
\text { [n (\%)] }\end{array}$ & & & \\
\hline \multicolumn{6}{|l|}{$\begin{array}{l}\text { Workstation objective assessment / Obiektywna ocena } \\
\text { stanowiska pracy }\end{array}$} \\
\hline \multicolumn{6}{|l|}{ work position / pozycja podczas pracy } \\
\hline sitting / siedząca & 975 & $512(52.5)$ & 0.191 & 1.000 & 1.000 \\
\hline alternate (standing/sitting) / zmienna (stojąca/siedząca) & 57 & $35(61.4)$ & & $0.695(0.402-1.202)$ & $0.557(0.291-1.064)$ \\
\hline \multicolumn{6}{|l|}{ desktop / biurko } \\
\hline in accordance with ISO / zgodne z ISO [24] ${ }^{\mathrm{a}}$ & 870 & $437(50.2)$ & $<0.001$ & 1.000 & 1.000 \\
\hline not in accordance with ISO / niezgodne z ISO [24] ${ }^{\mathrm{a}}$ & 162 & $110(67.9)$ & & $2.096(1.469-2.991)$ & $1.127(0.696-1.823)$ \\
\hline \multicolumn{6}{|l|}{ seat / krzesło } \\
\hline in accordance with ISO / zgodne z ISO [24] ${ }^{\mathrm{a}}$ & 911 & $467(51.3)$ & 0.002 & 1.000 & 1.000 \\
\hline not in accordance with ISO / niezgodne z ISO [24] ${ }^{\mathrm{a}}$ & 121 & $80(66.1)$ & & $1.855(1.246-2.763)$ & $1.698(1.117-2.581)$ \\
\hline \multicolumn{6}{|l|}{ footrest / podnóżek } \\
\hline in accordance with ISO / zgodne z ISO [24] ${ }^{a}$ & 772 & $382(49.5)$ & $<0.001$ & 1.000 & 1.000 \\
\hline not in accordance with ISO / niezgodne z ISO [24] ${ }^{a}$ & 260 & $165(63.5)$ & & $1.773(1.328-2.368)$ & $1.359(0.979-1.886)$ \\
\hline \multicolumn{6}{|l|}{$\begin{array}{l}\text { ISO requirements regarding the whole workstation / } \\
\text { wymagania ISO dotyczące całego stanowiska pracy [24] }\end{array}$} \\
\hline fulfilled / spełnione & 555 & $270(48.6)$ & 0.002 & 1.000 & 1.000 \\
\hline not fulfilled / niespełnione & 477 & $277(58.1)$ & & $1.462(1.143-1.871)$ & $2.375(1.124-5.018)$ \\
\hline \multicolumn{6}{|l|}{$\begin{array}{l}\text { Workstation subjective self-assessment / Subiektywna ocena } \\
\text { stanowiska pracy }\end{array}$} \\
\hline \multicolumn{6}{|l|}{ workstation as a whole / stanowisko jako całość } \\
\hline comfortable / wygodne & 855 & $410(48.0)$ & $<0.001$ & 1.000 & 1.000 \\
\hline uncomfortable / niewygodne & 177 & $137(77.4)$ & & $3.717(2.550-5.419)$ & $2.654(1.572-4.483)$ \\
\hline \multicolumn{6}{|l|}{ desktop / biurko } \\
\hline comfortable / wygodne & 889 & $434(48.8)$ & $<0.001$ & 1.000 & 1.000 \\
\hline uncomfortable / niewygodne & 143 & $113(79.0)$ & & $3.949(2.586-6.031)$ & $2.193(1.152-3.969)$ \\
\hline \multicolumn{6}{|l|}{ seat / krzesło } \\
\hline comfortable / wygodne & 884 & $478(54.1)$ & 0.093 & 1.000 & 1.000 \\
\hline uncomfortable / niewygodne & 148 & $69(46.6)$ & & $0.742(0.523-1.052)$ & $0.942(0.561-1.582)$ \\
\hline
\end{tabular}


age $\geq 50$ years old $(\mathrm{OR}=2.231,95 \% \mathrm{CI}: 1.236-4.026)$, body mass index (BMI) of $18.5-25 \mathrm{~kg} / \mathrm{m}^{2}(\mathrm{OR}=3.503$, 95\% CI: 2.047-5.995), and absence of regular physical activity (OR $=2.758,95 \%$ CI: 2.085-3.650).

Among the workstation elements, incomplete fulfillment of ISO 9241-5:1998 [24] standards was associated with a significant increased prevalence of MSDs $(\mathrm{OR}=1.462,95 \% \mathrm{CI}: 1.143-1.871)$, and the difference remained statistically significant when desktop, seat and footrest requirements were individually evaluated $(\mathrm{OR}=2.096,95 \% \mathrm{CI}: 1.469-2.991 ; \mathrm{OR}=1.855$, 95\% CI: $1.246-2.763$ and OR $=1.773,95 \%$ CI: $1.328-$ 2.368 , respectively). The sit-standing posture was also associated with increased prevalence of MSDs (61.4\% vs. $52.5 \%)$ but the difference was not statistically significant $(\mathrm{p}=0.191)($ Table 3$)$.
Similarly, subjective self-assessment identified an increased prevalence of complaints for subjects referring to their workplace as uncomfortable $(\mathrm{OR}=3.717$, 95\% CI: 2.550-5.419), in particular when a desktop was described as not comfortable $(\mathrm{OR}=3.949$, 95\% CI: 2.586-6.031) (Table 3).

Among job-content elements (Table 4), participants having a predominant task during the working shift referred to an increased prevalence for musculoskeletal disorders $(\mathrm{p}=0.055)$, in particular for activities associated with data entry $(\mathrm{p}<0.001)$ whereas factors such as external management of rest breaks $(\mathrm{p}=0.233)$, front-office activities $(\mathrm{p}=0.117)$, and even dissatisfaction towards current job $(\mathrm{p}=0.479)$ did not appear significantly associated with the MSDs diagnosis.

Table 4. Prevalence of musculoskeletal disorders (MSDs) in the office workers studied in Italy, 2012-2013, and their assessment of the job characteristics

Tabela 4. Występowanie zaburzeń mięśniowo-szkieletowych (MSDs) pracowników biurowych badanych we Włoszech w latach 2012-2013 a ich ocena parametrów pracy

\begin{tabular}{|c|c|c|c|c|c|}
\hline \multirow{2}{*}{$\begin{array}{l}\text { Variable } \\
\text { Zmienna }\end{array}$} & \multicolumn{2}{|c|}{$\begin{array}{l}\text { Respondends } \\
\text { Badani } \\
(\mathrm{N}=1032)\end{array}$} & \multirow{2}{*}{$\mathrm{p}$} & \multirow{2}{*}{ OR $(95 \% \mathrm{CI})$} & \multirow{2}{*}{$\mathrm{OR}_{\mathrm{a}}(95 \% \mathrm{CI})^{*}$} \\
\hline & $\begin{array}{c}\text { total } \\
\text { ogółem } \\
{[\mathrm{n}]}\end{array}$ & $\begin{array}{c}\text { with MSDs } \\
\text { z MSDs } \\
\text { [n (\%)] }\end{array}$ & & & \\
\hline \multicolumn{6}{|l|}{$\begin{array}{l}\text { Free management of rest breaks / Przerwy na odpoczynek } \\
\text { ustalane przez pracownika }\end{array}$} \\
\hline yes / tak & 897 & $469(52.3)$ & 0.233 & 1.000 & \\
\hline no / nie & 135 & $78(57.8)$ & & $1.249(0.866-1.800)$ & \\
\hline \multicolumn{6}{|l|}{$\begin{array}{l}\text { Job including front office / Praca wymagająca kontaktu } \\
\mathrm{z} \text { interesantami }\end{array}$} \\
\hline various tasks / różne czynności & 684 & $348(50.9)$ & ref. & 1.000 & 1.000 \\
\hline single task / jedna czynność & 348 & $199(57.2)$ & 0.055 & $1.290(0.994-1.672)$ & $1.360(1.010-1.831)$ \\
\hline data entry / wprowadzanie danych & 74 & $55(74.3)$ & $<0.001$ & $2.795(1.624-4.809)$ & $2.152(1.157-4.002)$ \\
\hline data processing / przetwarzanie danych & 37 & $21(56.8)$ & 0.487 & $1.267(0.650-2.470)$ & $1.203(0.823-1.758)$ \\
\hline word processing / przetwarzanie tekstów & 192 & $103(53.6)$ & 0.498 & $1.117(0.811-1.540)$ & $1.172(0.812-1.692)$ \\
\hline programming / programowanie & 45 & $20(44.4)$ & 0.404 & $0.772(0.421-1.417)$ & $1.033(0.493-2.163)$ \\
\hline \multicolumn{6}{|l|}{ Job satisfaction / Satysfakcja z pracy } \\
\hline $\begin{array}{l}\text { satisfied or highly satisfied / zadowolony lub bardzo } \\
\text { zadowolony }\end{array}$ & 471 & $244(51.8)$ & 0.479 & 1.000 & \\
\hline
\end{tabular}

Abbreviations as in Table 2 / Skróty jak w tabeli 2.

${ }^{*}$ As in Table 3 / Jak w tabeli 3. 


\section{Multivariate analysis}

Eventually, the logistic regression was modeled including sex, age, working age, BMI, physical activity, hours of VDU use/week. Adjusted estimate for global fulfillments of the ISO standards [24] requirements $\left(\mathrm{OR}_{\mathrm{a}}=2.375,95 \% \mathrm{CI}: 1.124-5.018\right)$, and in particular for the seat $\left(\mathrm{OR}_{\mathrm{a}}=1.698,95 \% \mathrm{CI}: 1.117-2.581\right)$ as well as subjective assessment of the workstation $\left(\mathrm{OR}_{\mathrm{a}}=2.654,95 \% \mathrm{CI}: 1.572-4.483\right)$ and desktop $\left(\mathrm{OR}_{\mathrm{a}}=2.193,95 \%\right.$ CI: 1.152-3.969) retained statistical significance. Eventually, $\mathrm{OR}_{\mathrm{a}}$ for job content suggested a significant association between musculoskeletal complaints and assignments including a single prevalent task $\left(\mathrm{OR}_{\mathrm{a}}=1.360,95 \% \mathrm{CI}: 1.010-1.831\right)$, confirming the increased prevalence for mainly data entry tasks $\left(\mathrm{OR}_{\mathrm{a}}=2.152\right.$, 95\% CI: 1.157-4.002) (Table 3 and 4).

\section{DISCUSSION}

The causal relationship between the development of WMSDs and computer related tasks has been disputed over decades but the contribution of physical and psychosocial factors to the development of musculoskeletal complaints is generally well accepted to have a strong evidence basis [1-4,10,16-20]. However, epidemiology of WMSDs remains still largely undefined, with large variations among countries [26-28]. Difference in incidence and prevalence rates may be caused by methodological differences across studies, particularly as far as the definition of symptomatic cases is concerned [26,29,30]: actually, many previous studies collected WMSDs and/or musculoskeletal complaints through questionnaires or similar self-assessments of participants $[18,28]$.

The main strengths of this study resides therefore in the assessment of MSDs and work environment, performed by the investigators at compulsory medical surveillance through a well defined case definition, and in the preliminary selection of study population, as we excluded from the study the subjects with a personal occupational history including exposure to occupational risk factors such as vibrations (either arm or whole-body), vibrating tools, weight lifting, repetitive and forceful movements. Moreover, subjects with underlying musculoskeletal diseases (i.e., rheumatoid arthritis, osteoarthritis and other disorders of the connective tissue) or severe trauma/ surgery, that had been referred to in terms of personal history, were similarly excluded. These inclusion criteria should collectively rule out main confound- ing risk factors not related with computer use in the pathogenesis of MSDs.

In this study population, prevalence for MSDs was 53\% and increased through age groups and peaked in the case of subjects $\geq 50$-year-old $(63.1 \%$, $\mathrm{OR}=2.231,95 \%$ CI: $1.236-4.026$ assuming 20-29-yearold group as the referent one) and of female sex (62.3\%, $\mathrm{OR}=2.832$, 95\% CI: 2.178-3.683). Among personal and job-related factors, working age $(\mathrm{OR}=1.934$, 95\% CI: $1.301-2.875$ and $\mathrm{OR}=2.223,95 \%$ CI: $1.510-3.271$ for groups of 10-14 years and $\geq 15$ years, respectively), the number of hours worked with a VDU per week $(\mathrm{OR}=1.537,95 \% \mathrm{CI}: 1.087-2.173)$ and the lack of regular physical activity (OR $=2.758,95 \%$ CI: 2.085-3.650) were associated with the prevalence of musculoskeletal complaints.

The lower risk for MSDs in the case of participants working $40 \mathrm{~h}$ or more per week may be explained both as a consequence of the reduced number of sampled subjects under this stratum (3.8\% of the study population) and because of the "healthy worker effect." In general, these results are therefore consistent with previous reports, and ultimately with the "effort-recovery model" [11]. As suggested by this model, MSDs would over time follow an imbalance between job demands and job resources, with increased mental and muscular fatigue leading to health complaints among employees not able to recover from the strain effects. In particular, sitting and working in awkward position for long hours, and performing repetitive manual tasks with high frequency, continuous low force demands on small muscle fibers, eventually would result in tissue damage [11,30,31].

Not coincidentally, in our study MSDs were more frequently identified among subjects whose workstations did not fulfill all the ISO standard 9241-5:1998 [24] requirements $\left(\mathrm{OR}_{\mathrm{a}}=2.375,95 \%\right.$ CI: 1.124-5.018), with a preeminent role for the chair $\left(\mathrm{OR}_{\mathrm{a}}=1.698,95 \% \mathrm{CI}: 1.117-2.581\right)$. Moreover, subjective assessment of the workstation was similarly well correlated with positive status for musculoskeletal complaints $\left(\mathrm{OR}_{\mathrm{a}}=2.654,95 \%\right.$ CI: $\left.1.1572-4.483\right)$.

Findings that suggest an increased prevalence for MSDs among participants prevalently performing data entry work $\left(\mathrm{OR}_{\mathrm{a}}=2.152,95 \% \mathrm{CI}\right.$ : $\left.1.157-4.002\right)$ are consistent both with previous reports and the aforementioned effort-recovery model. In fact, data entry involves very monotonous and repetitive tasks, simultaneously requiring a high degree of attention, and has been frequently described as both physically and psychologically demanding for VDU users [30-32]. 
However, other findings are not seemingly consistent with the effort-recovery model. First of all, prevalence of MSDSs was not significantly influenced by the rest breaks $(52.3 \%$ vs. $57.8 \%, \mathrm{p}=0.233)$ but these results may be explained by the study design. Previous reports actually proved a strong relationship between MSDs (in particular for the neck/shoulders) and the rest breaks focused on their dichotomous availability/unavailability. It is not a coincidence that a schedule of two $15 \mathrm{~min}$ breaks/day has become substantially conventional in all developed countries, which is why these results were subsequently disputed [28,30-32]. Due to the fact that Italian law about Health and Safety on the Workplaces (Legislative Decree No. 81, April 9, 2008 [22]) enforces compulsory rest breaks of 15 min every $2 \mathrm{~h}$ of continuous VDU use, we rather focused on their schedule management, accurate assessment of which was not performed. Eventually, the lack of further detail about rest breaks assessment is a significant limitation of this study.

Secondly, we found no significant positive effect for an alternate (standing/sitting) work posture as compared to conventional sitting posture $\left(\mathrm{OR}_{\mathrm{a}}=0.557\right.$, 95\% CI: 0.291-1.064). In this regard, despite the fact that some studies have identified sit-stand posture as more comfortable regarding musculoskeletal complaints, the workers usually have a lower level of usage compliance that may mitigate all benefits over time and face the lack of an arm support that may reduce the musculoskeletal strain for neck and upper arm $[28,31,32]$.

Thirdly, despite the fact that the contribution of psychosocial strain (e.g., high stress, high job demands, job strain and low co-worker support) to the development of MSDs has been diffusely acknowledged [27], factors such as front office assignments and dissatisfaction with the current job were not associated with a significantly higher prevalence of MSC (50.4\% vs. $55.3 \%, \mathrm{p}=0.117$ and $51.8 \%$ vs. $59.9 \%, \mathrm{p}=0.479$ ).

Eventually, several limitations should be addressed. First of all, our study recurred to a convenience sampling, including all workers from the parent companies participating in the original health survey, and ultimately the composition of the sample may not be representative of the Italian working population.

Moreover, our study lacked a detailed psychological assessment through specifically designed items, so we cannot rule out these results as the consequence of a study design ultimately lacking appropriate sensitivity, either.

Another limitation of this study resides in the exposure assessment as cumulative hours of exposure over a certain time span. More recent research suggests that more accurate evaluation of the exposure may be performed by recording the effective computer activity, and in particular keyboard strokes and mouse movements, including both average and peak exposure (i.e., number of beats/movements per minute) [30-33]. Unfortunately, not only may all these elements be of controversial interpretation but also data collection may be interpreted as contrary to the current Italian Labour Law (Law No. 300, May 20, 1970 [34]).

\section{CONCLUSIONS}

In our cross-sectional study, encompassing $1032 \mathrm{VDU}$ workers from Northern Italy, prevalence of MSDs was roughly similar to previous reports from developed and developing countries. Similarly, MSDs were associated with well known personal risk factors such as age, working age, lacking of physical activity. Ultimately, our study identified a significant effect of workstation design elements on MSDs prevalence.

In summary, these results not only reinforce the interpretation of MSDs among VDU workers through the effort-recovery model but also suggest that specific ergonomic requirements identified by current guidelines and international standards may be appropriate to reduce or prevent musculoskeletal symptoms among employees in the office environment.

\section{REFERENCES}

1. Wu S, He L, Li J, Wang J, Wang S. Visual display terminal use increases the prevalence and risk of work-related musculoskeletal disorders among Chinese office workers: A cross-sectional study. J Occup Health. 2012;54: 34-43, http://dx.doi.org/10.1539/joh.11-0119-OA.

2. Korhonen T, Ketola R, Toivonen R, Luukkonen R, Häkkänen $\mathrm{M}$, Viikari-Juntura E. Work related and individual predictors for incident neck pain among office employees working with video display units. Occup Environ Med. 2003;60:475-82, http://dx.doi.org/10.1136/oem.60.7.475.

3. Sillanpää J, Huikko S, Nyberg M, Kivi P, Laippala P, Uitti J. Effect of work with visual display units on musculoskeletal disorders in the office environment. Occup Med. 2003;53:443-51, http://dx.doi.org/10.1093/occmed/kqg120.

4. Wahlstrom J, Hagberg M, Toomingas A, Wigaeus Tornqvist E. Perceived muscular tension, job strain, physical exposure, and associations with neck pain among VDU users; a prospective cohort study. Occup Environ Med. 2004;61:523-8, http://dx.doi.org/10.1136/oem.2003.009563. 
5. European Agency for Safety and Health at Work. Workrelated musculoskeletal disorders: Prevention report. Luxembourg: Office for Official Publications of the European Communities; 2008.

6. Bongers PM, Ijmker S, van den Heuvel S, Blatter BM. Epidemiology of work related neck and upper limb problems: Psychosocial and personal risk factors (part I) and effective interventions from a bio-behavioural perspective (part II). J Occup Rehabil. 2006;16:279-302, http:// dx.doi.org/10.1007/s10926-006-9044-1.

7. Aarås A, Horgen G, Bjørset H-H, Ro O, Walsøe H. Musculoskeletal, visual and psychosocial stress in VDU operators before and after multidisciplinary ergonomic interventions. A 6 years prospective study - Part II. Appl Ergon. 2001;32:559-71, http://dx.doi.org/10.1016/S00036870(01)00030-8.

8. Juul-Kristensen B, Søgaard K, Strøyer J, Jensen C. Computer users' risk factors for developing shoulder, elbow and back symptoms. Scand J Work Environ Health. 2004;30:390-8, http://dx.doi.org/10.5271/sjweh.827.

9. Gerr F, Monteilh CP, Marcus M. Keyboard use and musculoskeletal outcomes among computer users. J Occup Rehabil. 2006;16:259-71, http://dx.doi.org/10. 1007/s10926-006-9037-0.

10. Eltayeb S, Staal JB, Hassan A, de Bie RA. Work related risk factors for neck, shoulder and arms complaints: A cohort study among Dutch computer office workers. J Occup Rehabil. 2009;19:315-22, http://dx.doi.org/10. 1007/s10926-009-9196-x.

11. Gawke JC, Gorgievski MJ, van der Linden D. Office work and complaints of the arms, neck and shoulders: The role of job characteristics, muscular tension and need for recovery. J Occup Health 2012;54:323-30, http://dx.doi. org/10.1539/joh.11-0152-OA.

12. Kaliniene G, Ustinaviciene R, Skemiene L, Januskevicius V. Associations between neck musculoskeletal complaints and work related factors among public service computer workers in Kaunas. Int Occup Med Environ Health. 2013;26: 670-81, http://dx.doi.org/10.2478/s13382-013-0141-z.

13. Huisstede BM, Wijnhoven HA, Bierma-Zeinstra SM, Koes BW, Verhaar JA, Picavet S. Prevalence and characteristics of complaints of the arm, neck, and/or shoulder (CANS) in the open population. Clin J Pain. 2008;24: 253-9, http://dx.doi.org/10.1097/AJP.0b013e318160a8b4.

14. Hooftman WE, van der Beek AJ, Bongers PM, van Mechelen $\mathrm{W}$. Is there a gender difference in the effect of workrelated physical and psychosocial risk factors on musculoskeletal symptoms and related sickness absence? Scand J Work Environ Health. 2009;35:85-95, http://dx.doi. org/10.5271/sjweh.1316.
15. Hooftman WE, van Poppel MN, van der Beek AJ, Bongers PM, van Mechelen W. Gender differences in the relations between work-related physical and psychosocial risk factors and musculoskeletal complaints. Scand J Work Environ Health. 2004;30:261-78, http://dx.doi. org/10.5271/sjweh.794.

16. Janwantanakul P, Pensri P, Jiamjarasrangsi W, Sinsongsook T. Associations between prevalence of self-reported musculoskeletal symptoms of the spine and biopsychosocial factors among office workers. J Occup Health. 2009;51:114-22, http://dx.doi.org/10.1539/joh.L8105.

17. Oha K, Animägi L, Pääsuke M, Coggon D, Merisalu E. Individual and work-related risk factors for musculoskeletal pain: A cross-sectional study among Estonian computer users. BMC Musculoskelet Disord. 2014;15:181, http://dx.doi.org/10.1186/1471-2474-15-181.

18. Wærsted M, Hanvold TN, Veiersted KB. Computer work and musculoskeletal disorders of the neck and upper extremity: A systematic review. BMC Musculoskelet Disord. 2010;11:79, http://dx.doi.org/10.1186/1471-2474-11-79.

19. Klussmann A, Gebhardt H, Liebers F, Rieger MA. Musculoskeletal symptoms of the upper extremities and the neck: A cross-sectional study on prevalence and symptom-predicting factors at visual display terminal (VDT) workstations. BMC Musculoskelet Disord. 2008;9:96, http://dx.doi.org/10.1186/1471-2474-9-96.

20. Cho C-Y, Hwang Y-S, Cherng R-J. Musculoskeletal symptoms and associated risk factors among office workers with high workload computer use. J Manipulative Physiol Ther. 2012;35:534-40, http://dx.doi.org/10.1016/ j.jmpt.2012.07.004.

21. Huisstede BMA, Miedema HS, Verhagen AP, Koes BW, Verhaar JAN. Multidisciplinary consensus on the terminology and classification of complaints of the arm, neck and/or shoulder. Occup Environ Med. 2007;64:313-9, http://dx.doi.org/10.1136/oem.2005.023861.

22. [The Act No. 81 of 9 April 2008 on health and safety in the workplaces. J Laws (GU) 2008, No. 101, ordinary suppl. No. 108]. Italian.

23. World Health Organization. Global recommendations on physical activity for health. Geneva: The Organization; 2010.

24. ISO 9241-5:1998. Ergonomic requirements for office work with visual display terminals (VDTs) - Part 5: Workstation layout and postural requirements. Geneva: International Organization for Standardization; 1998.

25. Occhipinti E, Colombini D, Molteni G, Menoni O, Boccardi S, Grieco A. [Development and validation of a questionnaire in the study of spinal changes in a working population]. Med Lav. 1988;79:390-42. Italian. 
26. Maakip I, Keegel T, Oakman J. Prevalence and predictors for musculoskeletal discomfort in Malaysian office workers: Investigating explanatory factors for a developing country. Appl Ergon. 2016;53(Pt A):252-7, http://dx.doi. org/10.1016/j.apergo.2015.10.008.

27. Andersen JH, Fallentin N, Thomsen JF, Mikkelsen S. Risk factors for neck and upper extremity disorders among computers users and the effect of interventions: An overview of systematic reviews. PLoS One. 2011;6(5):e19691, http://dx.doi.org/10.1371/journal.pone.0019691.

28. Sihawong R, Sitthipornvorakul E, Paksaichol A, Janwantanakul P. Predictors for chronic neck and low back pain in office workers: A 1-year prospective cohort study. J Occup Health. 2016;58(1):16-24, http://dx.doi.org/10.1539/ joh.15-0168-OA.

29. Riccò M, Cattani S, Signorelli C. Personal risk factors for carpal tunnel syndrome in female visual display unit workers. Int J Occup Med Environ Health. 2016;29(6): 927-936, http://dx.doi.org/10.13075/ijomeh.1896.00781.

30. Richter JM, van den Heuvel SG, Huysmans MA, van der Beek AJ, Blatter BM. Is peak exposure to computer use a risk factor for neck and upper-extremity symptoms? Scand J Work Environ Health. 2012;38:155-62, http://dx.doi.org/10.5271/sjweh.3196.

31. Davis KG, Kotowski SE. Postural variability: An effective way to reduce musculoskeletal discomfort in office work. Hum Factors. 2014;56:1249-61, http://dx.doi. org/10.1177/0018720814528003.

32. Rocha LE, Debert-Ribeiro M. Working conditions, visual fatigue, and mental health among systems analysts in São Paulo, Brazil. Occup Environ Med. 2004;61:24-32.

33. [The Act No. 300 of 20 May 1970 on the labour code. J Laws (GU) 1970, No. 131]. Italian.

This work is available in Open Access model and licensed under a Creative Commons Attribution-NonCommercial 3.0 Poland License / Ten utwór jest dostępny w modelu open access na licencji Creative Commons Uznanie autorstwa - Użycie niekomercyjne 3.0 Polska - http://creativecommons.org/ licenses/by-nc/3.0/pl/deed.en. 